\title{
Efficacy and quality of life assessment in the use of subcutaneous immunoglobulin treatment for children with primary immunodeficiency disorder
}

\author{
Department of Pediatric Immunology, Faculty of Medicine, Ege University, Izmir, Turkey
}

\author{
KEY WORDS \\ Subcutaneous immunoglobulin; \\ intravenous immunoglobulin; primary \\ immunodeficiency; quality of life; \\ pediatric immunology.

\section{Corresponding author} \\ Burcu Raziye Guven Bilgin \\ Ege University Children's Hospital \\ Department of Pediatric Immunology and \\ Rheumatology \\ Kazımdirik Mah \\ 35100 Bornova/İzmir, Turkey \\ E-mail: rzyburcuguven@hotmail.com
}

\section{Doi}

10.23822/EurAnnACI.1764-1489.179

\begin{abstract}
Summary
Introduction. Most patients with primary and secondary immunodeficiencies need regular intravenous immunoglobulin (IVIG) or subcutaneous immunoglobulin (SCIG) treatment. This study aimed to evaluate the serum IgG trough levels, frequency of mild and severe infections, frequency and duration of hospitalization, duration of absence of school, and quality of life in patients switching their IVIG therapy to SCIG administration. Materials. Twenty-nine patients with immunodeficiency on regular IVIG treatment and who agreed to receive SCIG treatment were included. Seven patients discontinued treatment after the first SCIG administration. We collected data regarding serum IgG levels, annual numbers of infections, hospital admissions, and adverse events prior to and following SCIG initiation. PedsQL tests such as scale total score (STS), physical health total score (PHTS), psychosocial health total score (PsyHTS), emotional functionality, social functionality, school/work problems score were calculated separately for all patients and their parents. Results. In twenty-two cases who were diagnosed as primary immunodeficiency, the most common indication for initiation of SCIG treatment was the long transfusion period of IVIG treatments and the difficulty of access to the hospital. No systemic side effects were noted except local redness, pain, and swelling on the injection site. The median IgG value was $588.9 \mathrm{mg} /$ dl during IVIG treatment and $872 \mathrm{mg} /$ dl one year after SCIG treatment. Annual frequency of infections and absence to school/work decreased significantly in the SCIG group while the annual number of hospitalizations and hospital stay time did not change significantly. There was a significant increase in the "quality of life" scores of the patients and their families. Conclusions. SCIG treatment provides ideal and protective immunoglobulin levels and offers the comfort of treatment in their home environment, thus increasing the patient's satisfaction and quality of life.
\end{abstract}

\section{Introduction}

Primary immunodeficiency diseases (PID) predispose patients to recurrent respiratory, skin, and gastrointestinal infections and require Immunoglobulin (Ig) replacement treatment (1). Immunoglobulin replacement therapy has been a standard treatment for PID for more than 50 years since Bruton and his colleagues treated a patient with agammaglobulinemia by using intramuscular gammaglobulin (2). Ig replacement treatment can be administered intravenously every 3-4 weeks or subcutaneously once every week. In 1991, Gardulf et al. demonstrated that subcutaneous immunoglobulin (SCIG) treatment was well tolerated and resulted in less systemic complications compared to intravenous immunoglobulin (IVIG) infusions in Swedish patients with PID (3). Recently, SCIG treatment has been widely used in Scandinavian countries, Europe, and other countries $(4,5)$. IVIG and SCIG treatment have different pharmacokinetics on serum IgG levels. IVIG treatment provides high peak IgG levels and low trough IgG levels, whereas SCIG treatment provides steady-state IgG levels (6). The two protocols have been tested 
and proven to be effective, safe, and well-tolerated. SCIG treatment has become popular in recent years because of its self-administration at home, resulting in more stable serum IgG levels, better protection against infections, and fewer complications. Chronicity of PID, delayed diagnosis, inadequate treatment and treatment side effects may effect the quality of life (QoL) of PID patients. QoL and the psychological impact of PID patients has been little studied in children. In a few previous studies, QoL was found to be poorer in PID patients than healthy controls $(7,8)$. Self infusion at home improves the QoL among patients, reduces travel time, and decreases healthcare system procedures $(9,10)$. In this study, we aimed to evaluate the levels of Immunoglobulin $G(\operatorname{IgG})$, frequency and severity of infections, frequency and duration of hospitalizations, time of absence from school/ work and QoL in PID patients receiving IVIG and then SCIG treatment.

\section{Patients and methods}

Twenty-nine patients in follow-up with the diagnosis of primary immunodeficiency at Ege University Department of Pediatric Immunology and switched to SCIG treatment between May 2015 and August 2018 were included in this retrospective study. Patients eligible to participate in the study were diagnosed according to the European Society for Immunodeficiencies (ESID) criteria and were receiving IVIG regularly. These patients had some problems related to IVIG treatment in the hospital such as difficulties with venous access, lengthy and expensive travels to reach the hospital, and economic burden. The other inclusion criteria were their consent to receive SCIG treatment at home. Patients who were receiving IVIG treatment for neurological diseases (such as chronic inflammatory demyelinating polyradiculoneuropathy), skin diseases (such autoimmune bullous skin disorders) and hematological problems (such as chronic immune trombocytopenia) were excluded.

Twenty-two patients $(75.8 \%)$ continued SCIG treatment, while seven patients $(24.2 \%)$ left the study after the first application. Twenty-three males (79.3\%), 6 females (21.7\%) patients were included in the beginning but 18 males $(81.8 \%), 4$ females (18.2\%) patients continued SCIG treatment. M/F ratio was 5/1, as usually seen in all PID patients (11). The diagnosis and results of molecular studies of all patients were listed in table $\mathbf{I}$. Medical records of patients were reviewed retrospectively. Age at first IVIG therapy, gender, diagnosis, IVIG treatment dose and frequency, systemic and local IVIG complications, serum IgG levels and complications of diseases (bronchiectasis, chronic sinusitis, etc.), frequency of mild and severe bacterial infections, frequency and duration of hospitalization, duration of absence from school/work and complications of IVIG treatment were recorded. Pneumonia, bronchitis, otitis media, sinusitis, arthritis, and septicemia were accepted as severe infections.
Age at first SCIG treatment, reasons for switching to SCIG therapy, SCIG dose, injection site, infusion rate, local and systemic adverse reactions were recorded in the SCIG group. Besides, IgG serum levels were recorded at the end of the second week and the $2^{\text {nd }}, 6^{\text {th }}$ and $12^{\text {th }}$ months. Patients were evaluated with the "quality of life test" Pediatric Quality of Life Inventory (PedsQL) on the $1^{\text {st }}$ day, $6^{\text {th }}$ and $12^{\text {th }}$ month of SCIG treatment. After one year of SCIG treatment, infections and their severity, duration of hospitalization, absence from school/work, and serum IgG levels were evaluated.

Patients who switched to SCIG treatment received the first dose 15 days after the last IVIG treatment. SCIG treatment dose was calculated by dividing the IVIG treatment dose into weekly doses. It was applied once a week or every ten days. Immunoglobulin preparations at a concentration of $10 \%$; $\mathrm{KIOVIG}^{\circledR}$ and GAMUNEX $^{\circledR}$ solutions were used, because there was no other concentrated licensed/approved immunoglobulin preparation in our country. The training nurse performed the first application of SCIG treatment, and the second and third applications were accompanied by the training nurse in the hospital and applied by the patient or parent. The patient/parent administered subsequent doses at home. SCIG treatment was applied to all patients with a soft set butterfly needle at a distance of $2-3 \mathrm{~cm}$ from the abdomen to the umbilicus. The total dose is divided into two equal doses from both sides of the abdomen per region; it was applied simultaneously with the manual rapid infusion technique with the help of injectors of maximum $25 \mathrm{cc}$ for patients less than $40 \mathrm{~kg}$, maximum $35 \mathrm{cc}$ for patients more than $40 \mathrm{~kg}$, with a speed of $2 \mathrm{ml} / \mathrm{min}$. A maximum of $10 \mathrm{~g}$ of immunoglobulin was applied in one application. No premedication was applied before infusions. Infusion times, application site and adverse effects were recorded.

Statistical analyzes were performed with SPSS 22.0 (IBM SPSS Statistics, Chicago, IL) program. Descriptive statistics incuded mean \pm standard deviation or median (minimum-maximum) for continuous variables and nominal variables as the number of cases and (\%). Chi-Square test for categorical data comparisons between groups, Kruskal-Wallis and Mann-Whitney U tests were used for comparison of numerical and ordinal data. Mann-Whitney $U$ test was used to evaluate the quality of life data. A p-value of $<0.05$ was considered statistically significant.

\section{Results}

Diagnostic distribution of patients with primary immunodeficiency were listed in table I, while common variable immunodeficiency (CVID) $(\mathrm{n}=13 ; 44.8 \%)$ and X-linked agammaglobulinemia (Bruton disease) $(\mathrm{n}=7 ; 24.1 \%)$ were the most common diseases. Five were diagnosed as Bruton's disease and two as CVID of the seven cases who preferred to discontinue SCIG treatment. The reasons to leave the treatment were mostly the 
concerns about injecting a drug to themselves or their children. Statistical analyses were performed on 22 patients who continued treatment. The age of the patients was significantly higher in the SCIG group (table II). The reasons for choosing SCIG treatment were difficulty for reaching the vascular access in 14 cases $(63.6 \%)$, frequent transfusion needs in 16 cases $(72.7 \%)$, long IVIG infusion time in 19 cases $(86.3 \%)$, difficulty in accessing the hospital in 18 cases $(81.8 \%)$. Five cases $(22.7 \%)$ had

Table I - Age, gender, molecular genetical findings and diagnosis of the study group.

\begin{tabular}{|c|c|c|c|c|}
\hline $\begin{array}{l}\text { Patient } \\
\text { No }\end{array}$ & $\begin{array}{l}\text { Age/ } \\
\text { Year }\end{array}$ & Gender & Diagnosis & Molecular and Genetics Diagnosis \\
\hline 1 & 23 & M & CVID & Not finalized yet \\
\hline 3 & 7.5 & M & TTC37 mutation & TTC37 gene: c.2210 T > C p.val 737A1a homozygous mutation was found \\
\hline 4 & 32 & M & Bruton disease & BTK gene; Glutamik asit 76 to stop (guanine358timin) \\
\hline 7 & 28 & M & CVID & $\begin{array}{c}\text { TNFSF13B gene (TACI); Pro97Pro ve Ser277Ser synonymous } \\
\text { amino acid mutations were found }\end{array}$ \\
\hline 8 & 14 & $\mathrm{~F}$ & CVID & Mutation was not found in TNGS (Targeted Next Generation sequencing test) \\
\hline 9 & 16 & M & CVID & $\begin{array}{l}\text {-TNFSF13 gene (APRIL); Asn96Ser mutation was found } \\
\text {-TNFSF13B gene (TACI): Thr27Thr synonymous amino acid mutation was found }\end{array}$ \\
\hline 12 & 22 & M & Activated PI3K-delta syndrome(APDS) & PIK3CD gene; c.970C > G p. Arg324Gly mutation was found \\
\hline 13 & 26 & M & CVID & TNFSF13B gene TACI: heterozygous c.204-205 insA (p.L69TfSX12) mutation was found \\
\hline 14 & 11 & M & LRBA deficiency & LRBA gene: c. $2496 \mathrm{C}>\mathrm{A}$, p.cys382Ter(P.c832*) mutation was found \\
\hline 15 & 13 & M & LRBA deficiency & LRBA gene: c. $2496 \mathrm{C}>\mathrm{A}$, p.cys $382 \operatorname{Ter}($ P.c832*) mutation was found \\
\hline 16 & 12 & $\mathrm{~F}$ & $\begin{array}{l}\text { HiperIgE syndrome } \\
\text { STAT } 3 \text { defect }\end{array}$ & STAT3 gene: $\mathrm{c} 114 \mathrm{C}>\mathrm{T}$,p.Arg382Tryp mutationu was found \\
\hline 17 & 7.5 & M & CVID & Mutation was not found in TNGS (Targeted Next Generation sequencing test) \\
\hline 18 & 19 & M & CVID & $\begin{array}{l}\text {-TNFSF13 gene (APRIL): Asn96Ser mutation was found } \\
\text {-TNFSF13B gene (TACI) : Thr27Thr synonymous amino acid mutation was found }\end{array}$ \\
\hline 19 & 12 & $\mathrm{~F}$ & CVID & Not finalized yet \\
\hline 24 & 5 & $\mathrm{~F}$ & CVID & PLCG2 gene; heterozygous p.ser718Arg mutation was found \\
\hline 25 & 8 & M & Bruton disease & BTK gene; c.36G > C p.Lys12Asn hemizygous mutation was found \\
\hline 26 & 3 & $\mathrm{~F}$ & Bruton disease & BTK gene; c.36G > C p.Lys12Asn hemizygous mutationu was found \\
\hline 27 & 1 & M & CVID & Not finalized yet \\
\hline 28 & 8 & M & Bruton disease & BTK gene; c.493T > G (p.Cys165Gly) hemizygous mutation was found \\
\hline 29 & 6 & M & Bruton disease & BTK gene; c.493T > G (p.Cys165Gly) hemizygous mutation was found \\
\hline
\end{tabular}

M: male; F: female. 
some personal preferences. None of the patients preferred SCIG treatment due to the serious side effects of IVIG treatment. There were some complications related to the underlying diseases in 21 patients. No complication was recorded for a CVID patient. Seven cases had only one complication, while the other 14 had two or more complications. Growth retardation $(\mathrm{n}=11 ; 50 \%)$, bronchiectasis $(\mathrm{n}=12 ; 54.5 \%)$, chronic sinusitis $(\mathrm{n}=3 ; 13.6 \%)$, hepatomegaly $(n=2 ; 9.0 \%)$, splenomegaly $(n=4 ; 18.0 \%)$, chronic diarrhea $(n=6 ; 27 \%)$, autoimmunity $(n=2 ; 9.0 \%)$, and some other complications $(\mathrm{n}=12 ; 54.5 \%)$ were observed.

\section{IVIG group}

Twenty-two patients in the study group received a median of $0.5(0.17-1) \mathrm{g} / \mathrm{kg}$ IVIG treatment in the last year before SCIG treatment. The median time between the two treatments was 45 (21-75) days. The median IgG value was 588.9 (136-1938) mg/ dl. Two patients with Hyper IgE syndrome and one patient with SH2D1A defect had hypergammaglobulinemia, so the mean IgG value was found to be slightly higher than age-related normal values (12). The median annual frequency of infections was 4 (1-12) times, severe bacterial infection was $0.5(0-3)$ times, and absence to school/work was found to be 12 (0-90) days. In IVIG study group, $9(37.5 \%)$ of 24 cases had rapid side effects such as fever and rash, while 15 cases (64.5\%) had no side effects. None of the cases had serious side effects due to IVIG treatment.

\section{SCIG group}

When switching to SCIG treatment, the monthly immunoglobulin dose received by the patients was divided into four, and a median of $0.11 \mathrm{~g} / \mathrm{kg} /$ week (0.06-0.41) immunoglobulin was administered subcutaneously. The average initial infusion time for SCIG therapy was 22.4 minutes (4-50 minutes). In all cases, the first three applications were performed by the training nurse, subsequent practices were performed by parents $(\mathrm{n}=15$; $68.1 \%)$ and by patients $(\mathrm{n}=7 ; 31.9 \%) .10 \%$ solutions were used in all cases for SCIG therapy: GAMUNEX ${ }^{\circledR}$ was preferred in 3 cases $(13.6 \%)$, and $\mathrm{KIOVIG}^{\circledR}$ in 19 cases $(86.4 \%)$. Local side effects (redness, swelling and mild pain) were observed in 20 cases $(90.9 \%)$. Systemic side effect due to SCIG treatment was not observed in any of the cases. Trough IgG levels after SCIG treatment were shown in figure 1. When IVIG treatment IgG trough levels were compared with SCIG trough levels at $12^{\text {th }}$ months, a statistically significant difference was found $(\mathrm{p}=$ 0.000) (table II). Annual frequency of infections, and absence to school/work decreased significantly in SCIG group while annual severe bacterial infections, number of hospitalizations, and hospital stay time did not change significantly (table II).

\section{Figure 1 - Trough IgG levels after SCIG treatment.}

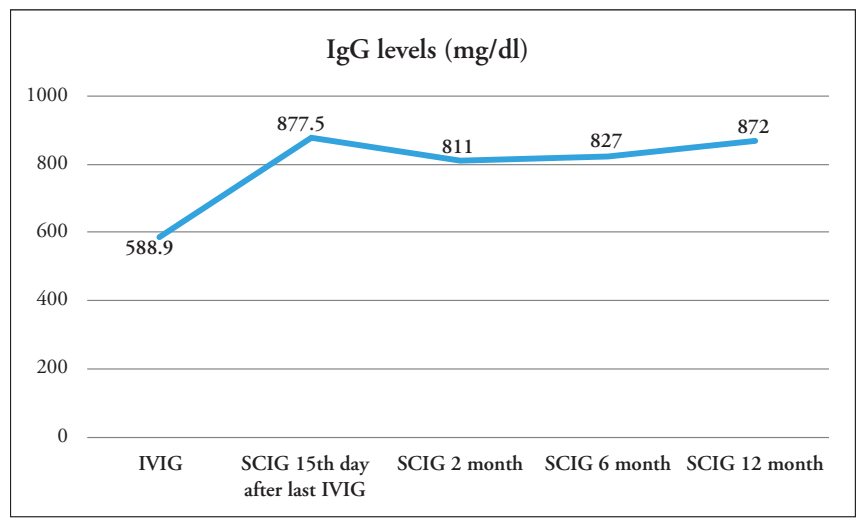

Table II - Clinical and laboratory features of patients during IVIG and SCIG treatments.

\begin{tabular}{|c|c|c|c|}
\hline Clinical and Laboratory Features & During IVIG Treatment & During SCIG Treatment & P values \\
\hline Age (years) median (min-max) & $5.5(1-16)$ & $11(4-30)$ & 0.000 \\
\hline Immunoglobulin dosage in the last year median (min-max) & $0.5(0.17-1)$ (g/kg/month) & $0.11(0.06-0.41)(\mathrm{g} / \mathrm{kg} /$ week $)$ & * \\
\hline $\begin{array}{l}\text { Time (days) between two immunoglobulin therapy medi- } \\
\text { an (min-max) }\end{array}$ & $45(21-75)$ & $7(7-10)$ & * \\
\hline $\operatorname{IgG}(\mathrm{mg} / \mathrm{dl})$ median (min-max) & $588.9(136-1938)$ & $872(411-2030)$ & 0.000 \\
\hline Annual frequency of infections (times) median (min-max) & $4(1-12)$ & $1(0-8)$ & 0.000 \\
\hline Annual severe bacterial infections (times) median (min-max) & $0.5(0-3)$ & $0(0-4)$ & 0.234 \\
\hline Number of hospitalizations in a year median (min-max) & $0(0-3)$ & $0(0-7)$ & 0.231 \\
\hline Annual hospital stay time (days) median (min-max) & $0(0-36)$ & $0(0-48)$ & 0.960 \\
\hline Absence to school/work days/year median (min-max) & $12(0-90)$ & $3(0-50)$ & 0.002 \\
\hline
\end{tabular}

*Parameters are not suitable for comparison. 
Details of PedsQL tests such as scale total score (STS), physical health total score (PHTS), psychosocial health total score (PsyHTS), emotional functionality, social functionality, school/work problems score which was carried out separately for 24 patients and their parents are shown in figures $\mathbf{2 , 3}$. These scores were determined three times during SCIG treatment and it was observed that the point value increased in each group. A statistically significant difference in the PedsQL scores $(\mathrm{p}<0.05)$ was found when the $6^{\text {th }}$ month and $12^{\text {th }}$ month were results compared with the first day.

\section{Discussion}

IVIG and SCIG have been repeatedly shown to reduce mild and severe infections and increase in serum IgG concentrations and are thus both approved methods of Ig replacement (13). In recent decades SCIG has been used increasingly, with some data showing increased IgG levels, improved patient quality of life indicators and decreased overall cost to the healthcare system when compared to IVIG (13).

In the follow-up of immunoglobulin treatments, the frequency of mild and severe infections, time lost from school and work, and serum trough $\operatorname{IgG}$ levels are critical indicators to determine the efficacy of the treatment. Although the target serum IgG level is stated as at least $500 \mathrm{mg} / \mathrm{dl}$ in some publications, it has been observed that $\operatorname{IgG}$ dosage needs to be adjusted on an individual basis to prevent infections $(14,15)$. According to the data obtained from recent studies, the recommended average serum IgG value has to be $700-800 \mathrm{mg} / \mathrm{dl}$ (16). In a prospective study $(\mathrm{n}=60)$ by Gardulf et al. (17), SCIG therapy for the first 15 weeks of treatment is defined as 'washout period' and 16-43 weeks as "efficacy period". In the same study, while the mean serum IgG level was $780 \mathrm{mg} / \mathrm{dl}$ at the beginning of the treatment in the pediatric age group, the mean of the efficacy

Figure 2 - Patients' PedsQL test results.

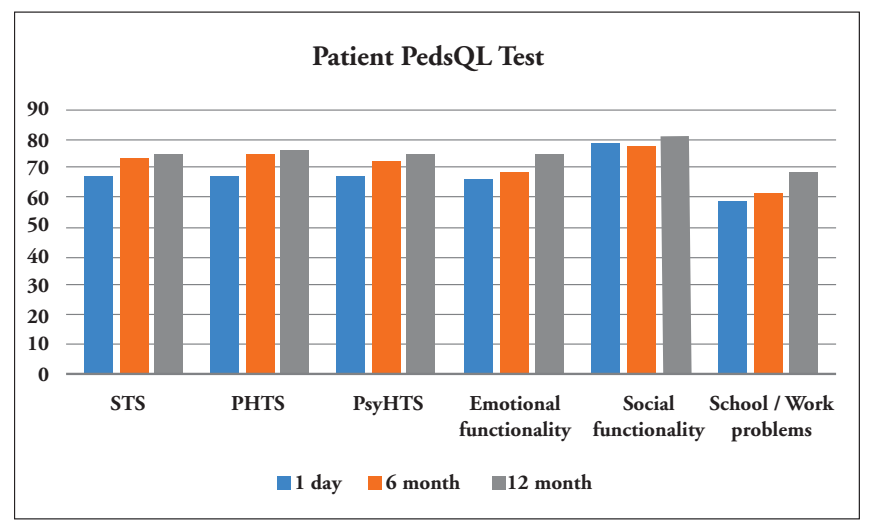

STS: scale total score; PHTS: physical health total score; PsyHTS: psychosocial health total score. phase increased to $920 \mathrm{mg} / \mathrm{dl}$. In a study by Chapel et al. (18) $(\mathrm{n}=40)$, it was reported that the mean serum trough value of IVIG treatment was $780-840 \mathrm{mg} / \mathrm{dl}$ while it was $800-910 \mathrm{mg} /$ $\mathrm{dl}$ in SCIG treatment. In the study by Bezrodnik et al. (19) (n = 15), the mean serum IgG value was $960 \mathrm{mg} / \mathrm{dl}$ before SCIG treatment, while it was reported to be $1309 \mathrm{mg} / \mathrm{dl}$ at $24^{\text {th }}$ week and $1230 \mathrm{mg} / \mathrm{dl}$ at $36^{\text {th }}$ week. In the study of Aydiner et al. (20) (n:16), mean serum IgG $976 \mathrm{mg} / \mathrm{dl}$ increased to $1025 \mathrm{mg} /$ $\mathrm{dl}$ after treatment, but no statistically significant difference was found. In our study, while the median trough IgG levels were $588.9 \mathrm{mg} / \mathrm{dl}$ before SCIG treatment, it was found to be 827 $\mathrm{mg} / \mathrm{dl}$ in the 6th month of the treatment and $872 \mathrm{mg} / \mathrm{dl}$ in the $12^{\text {th }}$ month, showing that they reached the recommended level. Patients with PID most frequently present with sinopulmonary findings. In previous reports, the number of mild infections, such as upper respiratory tract infections, decreased significantly under SCIG treatment. However, when the authors compared the frequency of acute severe bacterial infections between the two treatments, it was shown that there was no statistically significant difference $(17,21)$. In our study, we found a significant decrease in the annual frequency of infections in the SCIG group, while annual severe bacterial infections did not change significantly (table II). Our findings on the frequency of infections were consistent with the results in the literature.

The patient or the parent can administer SCIG treatment at home, and this treatment reduces dependency on the hospital and days off school and work due to illness (22). Besides, it does not require vascular access; this highly increases the life quality of the patients. In our study, absence from school/work decreased significantly in the SCIG group (median three days) compared to the IVIG group (median 12 days) (table II). How-

Figure 3 - Parents' PedsQL test results.

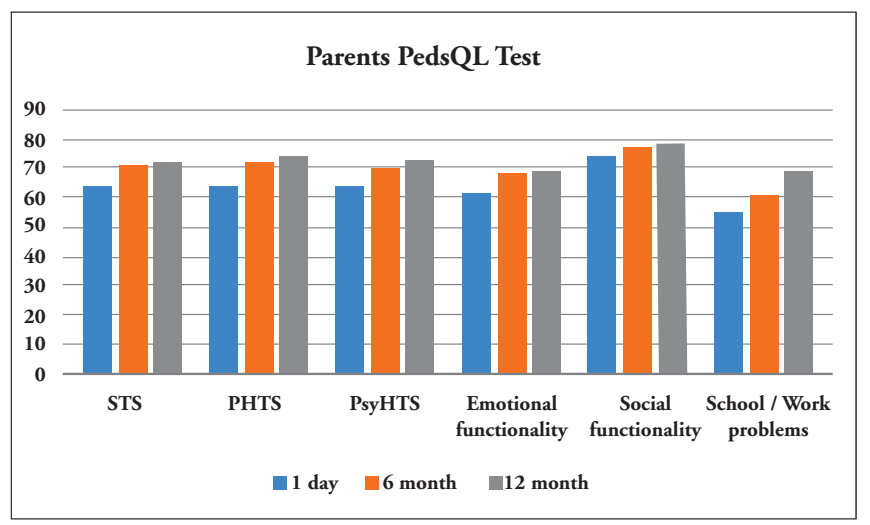

STS: scale total score; PHTS: physical health total score; PsyHTS: psychosocial health total score. 
ever, the number of hospitalizations or duration of stay in hospital did not change significantly between the groups (table II). Health-related Qol in PID patients was measured in limited studies. Ateinia et al. (8) measured QoL using PedsQL and SF-36 questionnaries in 70 PID patients. Patients expressed reduced scores in some mental and physical components (8). Patients with long follow-up time had higher scores in mental components and still low scores in physical components (8). Titman et al. (7) collected both parental and child ratings using standardised questionnaries such as PedsQL and SDQ in PID patients. Nineteen children and 43 parents completed the SDQ and 39 children and 43 parents completed the PedsQL. Higher rates of psychological difficulties were found in PID cases when compared with healthy controls (7). QoL was also poorer than in healthy controls. These QoL studies were also performed after switching IVIG treatments to SCIG and SCIG treatment has been found to improve QoL (23). In the study by Vultaggio et al. (24) ( $\mathrm{n}=50)$; while there was no significant difference in physical, daily activity and total health scores, there was a significant improvement in the QoL index. In a study by Gardulf et al. (25), the author emphasized the increase in the autonomy and QoL of the patient's self-application at home and being connected to others. In our study, the QoL including physical health score, psychological health score and total health score, emotional functionality, social functionality, school/ work life was found to be increased in both patients and families. It has been reported that side effects during IVIG therapy were observed in $20 \%$ of cases (26). Although most of these are temporary and non-serious side effects, $2-6 \%$ of them are serious (27). Side effects due to IVIG treatment are observed in 3 different groups; rapid side effects (within 6 hours after infusion), delayed side effects (within 6 hours, 1 week after infusion) and late side effects (weeks and months later) (28). In our study, 9 (37.5\%) of 24 cases had rapid side effects such as fever and rash, while 15 cases $(64.5 \%)$ had no side effects. None of the cases in study group had serious side effects due to IVIG treatment. While IVIG directly enters the blood, SCIG first passes into the lymphatic circulation and then into the bloodstream with the thoracic duct. Therefore, in SCIG, the slow transition to systemic circulation increases the tolerability of SCIG and reduces the possibility of systemic side effects (29). Although systemic side effects due to SCIG treatment are rare, local side effects such as rash, itching, and mild pain are observed. In the study by Chapel et al. (18) (n = 30), local side effects such as pain, redness and swelling were observed in all cases, while systemic side effects were observed only in 3.3\%. In another study by Bezrodrik et al. (19) ( $\mathrm{n}=15)$, 12 cases reported swelling, redness, pain and itching, while no systemic side effects were reported. In the study by Aydiner $e t a l$. (20) , 6\% of local side effects (redness itching, swelling) were reported, while systemic side effects were not reported. In our study, while more local side effects $(\mathrm{n}=22)(91.6 \%)$ (redness, swelling, mild pain) due to SCIG application developed, no systemic side effects were observed, similar to other studies. It is thought that the presence of more local side effects is due to the rapid push and short SCIG infusion time (27.4 \pm 17.7 minutes).

SCIG treatment has many advantages for patients who do not want to be hospitalized. These patients have difficulty for the venous vascular access or they cannot tolerate IVIG treatment or they do not want to stay in the hospital for a long time and want a more flexible treatment program (30). It is preferred also in terms of increasing the QoL of the patients who live far away from the hospital and who have busy work and school life and travel frequently (31). In the studies by Chouksey et al. (32) and Aydiner et al. (33); it has been reported that the most common reason to prefer SCIG treatment is difficulty in reaching the vascular access and side effects due to IVIG treatment. On the other hand, some patients may be discouraged from switching from IVIG to SCIG on account of perceived inconvenience, concerns about adverse effects at home and fear of needle sticks (33). In our study, the most frequent reasons were the long IVIG infusion period in the hospital and the difficulty to travel to the hospital. This situation can be explained by the fact that the pediatric immunology clinic of our hospital is one of the largest pediatric immunology clinics in the Aegean region and the number of patients living far away is very high.

The recommended starting dose for the treatment of Immunoglobulin G is for IVIG; $0.4-0.6 \mathrm{~g} / \mathrm{kg}$ in 3 or 4 week intervals, 0.1 $1.15 \mathrm{~g} / \mathrm{kg}$ week for SCIG (29). There are differences between Europe and America in terms of dose applied in the transition from IVIG treatment to SCIG treatment. In European countries, the amount of IgG given monthly is divided into 4-week doses at the same rate and administered subcutaneously (34). The American Food and Drug Administration (FDA) recommends a weekly dose calculation by multiplying the current dose by changing the coefficients depending on the product concentration $(\times 1.37$ for $16 \%$ and 1.53 for $20 \%$ products) (27). In our study, the median IVIG dose was $0.5 \mathrm{~g} / \mathrm{kg}$ and it was similar to the literature. The SCIG doses taken by the patients were administered by dividing them evenly into weekly doses. In our study, the median SCIG dose was $0.11 \mathrm{~g} / \mathrm{kg} /$ week and it was consistent with the literature data.

Application of SCIG at high volume and speed is known to increase the development of local side effects such as swelling, redness and pain. Recent, studies have shown that the application of $35 \mathrm{ml} / \mathrm{h}$ does not create any more side effects than the application of $20 \mathrm{ml} / \mathrm{h}$ (35). In our study, no more than $100 \mathrm{ml}$ was administered once and no more than $50 \mathrm{ml}$ per region was administered in 18 minutes. The average initial infusion time was $22.4 \mathrm{~min}-$ utes (4-50 minutes), and at the end of one year, the average infusion time extended to 26.7 minutes (12-75 minutes). Similar to the work of Vultaggio et al. (34), in our study local side effects were observed to decrease when the infusion time increased in cases with ongoing and home applications.

There are two limitations of this study. First, the study is limited by the small cohort size and secondly by short term study dura- 
tion (3 years). If we had longer follow-up time, we could decide about the benefits of SCIG therapy on the disease complications occured before and following the improvements in patients' disease complications, quality of life indexes might be much better.

\section{Conclusions}

In conclusion, SCIG treatment can be preferred in cases followed-up with the diagnosis of primary and secondary immunodeficiency due to difficulties in access to the venous vein, serious side effects due to IVIG, difficulty in reaching to hospital and also for the patients who desire a more flexible lifestyle. In addition, in patients using IVIG and with very low serum trough IgG value, SCIG is a good treatment option. We suggest that administration of smaller doses in shorter periods increases the serum trough IgG level and provides a more stable level, which is due to pharmacokinetic and dynamic differences. SCIG treatment reduces abcence from school/ work and provides time for people to travel comfortably and to move freely, due to the decrease in the number of infections and their application at home resulting an increase in the quality of life of both the patients and the families.

\section{Conflict of interests}

The authors declare that they have no conflict of interests.

\section{References}

1. Busse PJ, Razvi S, Cunningham-Rundles C. Efficacy of intravenous immunoglobulin in the prevention of pneumonia in patients with common variable immunodeficiency. J Allergy Clin Immunol 2002;109(6):1001-4.

2. Buckley CR. Agammaglobulinemia, by Col. Ogden C. Bruton, MC, USA, Pediatrics, 1952;9:722-728. Pediatrics 1998;102(1 Pt 2):213-5.

3. Gardulf A, Hammarstrom L, Smith CI. Home treatment of hypogammaglobulinaemia with subcutaneous gammaglobulin by rapid infusion. Lancet 1991;338(8760):162-6.

4. Gaspar J, Gerritsen B, Jones A. Immunoglobulin replacement treatment by rapid subcutaneous infusion. Arch Dis Child 1998;79(1):48-51.

5. Quinti I, Pierdominici M, Marziali M, et al. European surveillance of immunoglobulin safety-Results of initial survey of 1243 patients with primary immunodeficiencies in 16 countries. Clin Immunol 2002;104(3):231-6.

6. Ballow M. Optimizing immunoglobulin treatment for patients with primary immunodeficiency disease to prevent pneumonia and infection incidence: Review of the current data. Ann Allergy Asthma Immunol 2013;111(6 Suppl):S2-5.

7. Titman P, Alwood Z, Gilmour C, Malcolmson C, Duran-Persson C, Cale C, Davies G, Gaspar H, Jones A. Quality of life in children with primary antibody deficiency. J Clin Immunol 2014;34: 844-52.

8. Ateinia B, Montazeri A, Tavakol M, et al. Measurement of health-related quality of life in primary antibody-deficient patients. Immunological Invest 2017;46:329-40.

9. Nicolay U, Kiessling P, Berger M, et al. Health-related quality of life and treatment satisfaction in North American patients with primary immunedeficiency diseases receiving subcutaneous IgG self-infusions at home. J Clin Immunol 2006;26(1):65-72.

10. Gardulf A, Nicolay U. Replacement IgG therapy and self-therapy at home improve the health-related quality of life in patients with primary antibody deficiencies. Curr Opin Allergy Clin Immunol 2006;6(6):434-42.

11. Bonilla FA, Khan DA, Ballas ZK, Chinen J, Frank MM,Hsu JT. Practice parameter for the diagnosis and management of primary immunodeficiency. J Allergy Clin Immunol 2015;91:1186-99.

12. Aksu G, Genel F, Koturoğlu G, Kurugöl Z, Kutukculer N. Serum immunoglobuline (IgG, IgM, IgA) and IgG subclass concentrations in healthy children: a study using nephelometric technique. Turk J Pediatr 2006;48:19-24.

13. Walter G, Kalicinsky C, Warrington R, Miguel M, Reyes J, Rubin TS. Delivery of subcutaneous immunoglobin by rapid push infusion for primary immunodeficiency patients in Manitoba: a retrospective review. Allergy Asthma Clin Immunol 2020;16:34-3.

14. Jolles S, Orange JS, Gardulf A, et al. Current treatment options with immunoglobulin $G$ for the individualization of care in patients with primary immunodeficiency disease. Clin Exp Immunol 2015;179(2):146-60.

15. Pecoraro A, Ricci S, Vultaggio A, Boggia GM, Spadaro G. Correlations among subcutaneous immunoglobulin dosage, immunoglobulin $\mathrm{G}$ serum pre-infusional levels and body mass index in primary antibody deficiency patients: A pooled analysis from the SHIFT/ IBIS studies. Clinical Drug Investigation 2020;40:279-86.

16. Orange JS, Belohradsky BH, Berger M, et al. Evaluation of correlation between dose and clinical outcomes in subcutaneous immunoglobulin replacement therapy. Clin Exp Immunol 2012;169(2):172-81.

17. Gardulf A, Nicolay U. Replacement IgG therapy and self-therapy at home improve the health-related quality of life in patients with primary antibody deficiencies. Curr Opin Allergy Clin Immunol 2006;6(6):434-42.

18. Chapel HM, Spickett GP, Ericson D, Engl W, Eibl MM, Bjorkander J. The comparison of the efficacy and safety of intravenous versus subcutaneous immunoglobulin replacement therapy. J Clin Immunol 2000;20(2):94-100.

19. Bezrodnik L, Gómez Raccio A, Belardinelli G, et al. Comparative study of subcutaneous versus intravenous IgG replacement therapy in pediatric patients with primary immunodeficiency diseases: A multicenter study in Argentina. J Clin Immunol 2013;33(7):1216-22.

20. Aydıner EK, Kıykım A, Barış S, Özen A, Barlan I. Use of subcutaneous immunoglobulin in primary immune deficiencies. Turk Pediatr Ars 2016;51(1):8-14.

21. Bonagura VR, Marchlewski R, Cox A, Rosenthal DW. Biologic $\mathrm{IgG}$ level in primary immunodeficiency disease: The IgG level that protects against recurrent infection. J Allergy Clin Immunol 2008;122(1):210-2.

22. Windegger TM, Nghiem S, Nguyen K, Fung YL, Scuffham PA. Primary immunodeficiency disease: a cost-utility analysis comparing intravenous vs subcutaneous immunoglobulin replacement therapy in Australia. Blood Transfus 2020;18:96-105.

23. Samaan K, Levasseur MC, Decaluwe H, et al. SCIg vs IVIg: Let's Give Patients the Choice! J Clin Immunol 2014;34(6):611-4.

24. Vultaggio A, Azzari C, Milito C, et al. Subcutaneous Immunoglobulin Replacement Therapy in Patients with Primary Immunodeficiency in Routine Clinical Practice: The VISPO Prospective Multicenter Study. Clin Drug Investig 2015;35(3):179-85. 
25. Gardulf A, Nicolay U, Math D, et al. Children and adults with primary antibody deficiencies gain quality of life by subcutaneous IgG self-infusions at home. J Allergy Clin Immunol 2004;114(4):936-42.

26. Pierce LR, Jain N. Risks Associated With the Use of Intravenous Immunoglobulin. Transfus Med Rev 2003;17(4):241-51.

27. Orange JS, Hossny EM, Weiler CR, et al. Use of intravenous immunoglobulin in human disease: A review of evidence by members of the Primary Immunodeficiency Committee of the American Academy of Allergy, Asthma and Immunology. J Allergy Clin Immunol 2006;117(4 Suppl):S525-53.

28. Stiehm ER. Adverse effects of human immunoglobulin therapy. Transfus Med Rev 2013;27(3):171-8.

29. Berger M. Choices in IgG replacement therapy for primary immune deficiency diseases: Subcutaneous IgG vs. intravenous IgG and selecting an optimal dose. Curr Opin Allergy Clin Immunol 2011;11(6):532-8.

30. Berger M. Subcutaneous immunoglobulin replacement in primary immunodeficiencies. Clin Immunol 2004;112(1):1-7.
31. Shapiro RS. Subcutaneous immunoglobulin therapy given by subcutaneous rapid push vs infusion pump: A retrospective analysis. Ann Allergy Asthma Immunol 2013;111(1):51-5.

32. Chouksey A, Duff K, Wasserbauer N, Berger M. Subcutaneous immunoglobulin $\mathrm{G}$ replacement therapy with preparations currently available in the United States for intravenous or intramuscular use: Reasons and regimens. Allergy Asthma Clin Immunol 2005;1(3):120-30.

33. Mallick R, Henderson T, Lahue BJL, Kafal A, Bassett P, Scalchunes C. Subcutaneous immunoglobulin in primary immunodeficiency-impact of training and infusion characteristics on patient-reported outcomes. BMC Immunology 2020;21:47-57.

34. Hoffmann F, Grimbacher B, Thiel J, et al. Home-based subcutaneous immunoglobulin $\mathrm{G}$ replacement therapy under real-life conditions in children and adults with antibody deficiency. Eur J Med Res 2010;15(6):238-45.

35. Hansen S, Gustafson R, Smith CI, Gardulf A. Express subcutaneous IgG infusions: Decreased time of delivery with maintained safety. Clin Immunol 2002;104(3):237-41. 